\title{
MICROFABRICATED TORSIONAL ACTUATOR USING SELF-ALIGNED PLASTIC DEFORMATION
}

\author{
Jongbaeg Kim, Hyuck Choo, Liwei Lin and Richard S. Muller \\ Berkeley Sensor \& Actuator Center \\ University of California, Berkeley
}

\begin{abstract}
We describe microfabricated torsional actuators that are made using self-aligned plastic deformation in a batch process. The microactuators are formed in single-crystal silicon and driven by vertical comb-drives. Structures have been built that resonate at frequencies between 1.90 and $5.33 \mathrm{kHz}$ achieving scanning angles up to 19.2 degrees with driving voltages of $40 \mathrm{~V}_{\mathrm{dc}}$ plus $13 \mathrm{~V}_{\mathrm{ac}}$. After continuous testing of 5 billion cycles at the maximum scanning angle, there appears to be no observable degradation or fatigue of the plastically deformed silicon torsion bars. We present measured results obtained with MEMS scanning mirrors; the actuators may be useful for many other MEMS applications.
\end{abstract}

\section{INTRODUCTION}

Electrostatic comb-drive actuatôrs are used in numerous MEMS applications where they have demonstrated their capability for extended ranges-of-movement, stable and reliable operation, and design flexibility in different frequency ranges. In particular, many optical MEMS applications employ comb-drive actuators for torsional motions. For example, lateral comb-drives and mechanical hinges or linkages made of polysilicon [1] or single-crystal silicon [2] have been demonstrated to make torsional actuators. From these applications, linkage- and hinge- designs have been identified as sources for reliability problems and for limitations on maximum frequencies of operation. One design for torsional actuators uses vertically aligned comb-drives to achieve both higher frequencies and larger scan angles than those characterizing the planar polysilicon structures [3]. Other vertically aligned comb drives have employed polysilicon on SOI [4] and single-crystal silicon fabricated using wafer bonding [5]. However, these devices commonly face the requirement of placing two sets of comb structures at different vertical positions for operation, in which alignment can be problematic. Although a self-aligned vertical comb has also been demonstrated [6], it is fabricated using wafer bonding, grinding, polishing and silicon anisotropic-etching processes.

While all of the vertical-comb actuators described above are SVC type (Staggered Vertical Comb-drive), the AVC type (Angular Vertical Comb-drive) actuators have been reported recently to have $50 \%$ more torsional displacement than the SVC types when the comb geometries are equivalent $[7,8]$. The basic fabrication processes for AVCs are first to define the stationary and movable comb structures on the same level of silicon layer, and to deflect either the stationary comb structure by the residual stress induced by a metal layer [7], or else the movable comb structure using the surface-tension force that arises as a result of the reflow of a patternedphotoresist layer [8]. A limitation of the residual-stress method of fabrication is that the structure must be sufficiently flexible so that it undergoes appreciable deformation. Designs that meet this requirement are generally limited in their ultimate operating frequencies to a few hundreds $\mathrm{Hz}$ [7]. The challenging problems for the reflow of polymer hinges have been the control and reliability of the polymer material.

To improve the performance of torsion-bar microactuators, AVC-type torsional actuators that are composed of all-single-crystal-silicon structures have been fabricated using controlled-plastic-deformation in silicon that is annealed at elevated temperatures [9]. In recent years, polysilicon [10] and single-crystal silicon [11] membranes were demonstrated to be plastically deformed forming hemispherical domed structures as a result of the pressures of heated gases trapped in a cavity. Plastically deformed polysilicon structures have been used in a self-assembled MEMS process [12] and, on a meso-scale for silicon-chip mounting [13]. We report a controlled, repeatable batch process based on the plastic deformation of silicon to make torsional microactuators, describing the fabrication process and the dynamic performance of the acutuators which are demonstrated in their application to scanning micromirrors.

\section{DESIGN AND FABRICATION}

The design and fabrication of torsion-bar-supported scanning mirrors are demonstrated as an example of verticalcomb actuators made by the plastic-deformation process. Figure 1 shows the new process. A torsion-bar-supported plate (which may, for example, function as a mirror) is formed in a first wafer. Using DRIE, projecting pillars are configured in a second wafer and positioned such that, when the two wafers are stacked together, the projecting pillars push on and depress the mirrors on the first wafer. The two stacked wafers are annealed at temperatures greater than $800^{\circ} \mathrm{C}$ causing the torsion bar to be plastically strained so that, after cooling to room temperature and separating the two wafers, the mirrors are permanently tilted from their original planar positions.

To start the process, the top- and bottom-combs and mirror plates are initially defined on the same device layer of an SOI wafer in Fig.1 (a) (c). During the DRIE (Deep Reactive Ion Etching) step, not only the actuator itself, but also the depressions are formed. The backside etching of the substrate wafer underneath the mirror and combs removes sufficient silicon to provide clearance for the mirror motion. This backside alignment is not critical and even tens of microns of alignment errors are acceptable. A second wafer is processed with protrusions that align to depressions in the comb-actuator wafer. A second wafer is configured with pillars that displace the comb structures to the desired tilt angles. The two mated wafers are annealed in a furnace at $800 \sim 900^{\circ} \mathrm{C}$. When they are separated, the comb actuators are plastically deformed as shown in Fig. 1(f). Throughout the whole process, no critical alignment is necessary. Top and bottom combs are self-aligned because they are defined in a 
single masking step, and device and lid wafers are also selfaligned due to the depressions and protrusions.

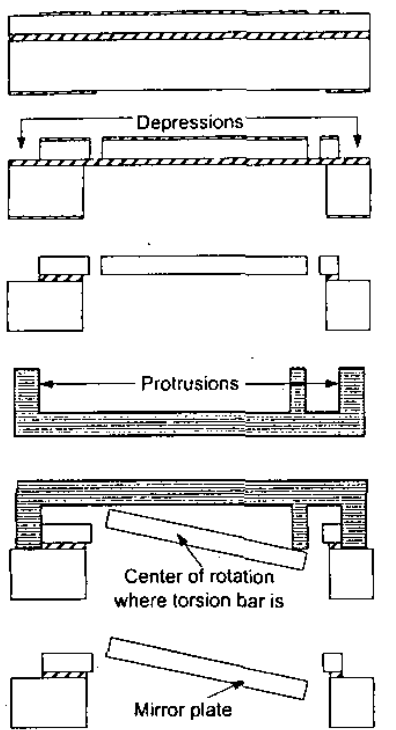

(a) Pattern the thermal oxide layers on the top and bottom surfaces of the SOI wafer.

(b) DRIE the device and handle layers of the SOI wafer.

(c) Removed the therma oxide layers as well as the exposed buried oxide layer.

(d) Create the pillar structures on the lid wafer by DRIE.

(e) Place the lid wafer on top of the device wafer and heat the stacked wafers above the glass transition temperature of silicon in the furnace.

(f) Remove the lid wafer.

Figure 1. Process flow for plastically deformed vertical actuators

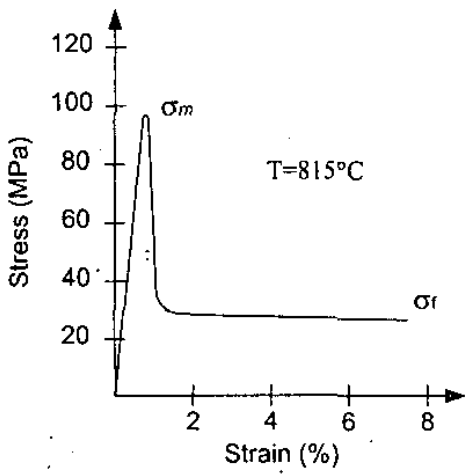

Figure 2. Stress-strain curve of silicon at an elevated temperature (from [9], [11])

in figure 2, a typical stress-strain curve of single -crystal silicon at high temperature is shown $[9,11]$. At room temperature, silicon is specified as a brittle material with a yield stress of $\sim 600 \mathrm{MPa}$. At an elevated temperature, the mechanical properties of silicon change dramatically. The maximum yield stress $\left(\sigma_{m}\right)$ decreases due to the increased mobility of dislocations in the crystal. At a temperature above $600^{\circ} \mathrm{C}$, the structures begin to plastically deform with a much reduced flow stress ( $\sigma_{r}$ : the stress needed to continue plastic deformation) instead of fracture when the induced stress in the silicon structure exceeds the yielding stress. When the pillar structure on the lid wafer pushes one edge of the mirror surface, the mirror and moving combs rotate on the torsional springs inducing stresses greater than $\sigma_{\mathrm{m}}$ in the springs by elastic deformation. Stresses are also induced in the mirror plate; however, the magnitude of the stress in the mirror is much smaller than $\sigma_{m}$ or $\sigma_{f}$ according to finite-element analysis and negligible compared to that in the torsional springs. Once the temperature is increased, the stresses in the torsional springs are relieved causing their plastic deformation. No deformation of the mirror plate is observed for the annealing temperature of $800 \sim 900^{\circ} \mathrm{C}$.

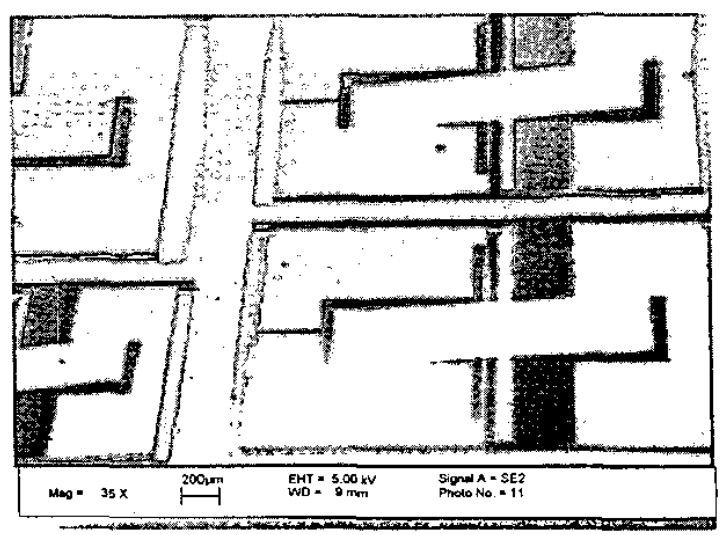

Figure 3. SEM picture of batch-processed scanning mirrors using plastic deformation of silicon

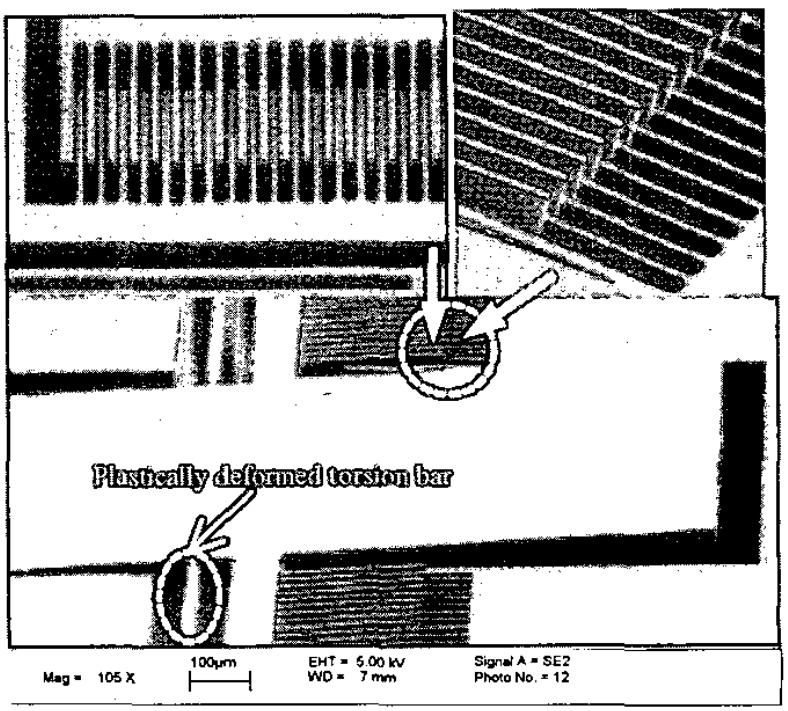

Figure 4. Thermo-plastically tilted mirror and precisely aligned vertical combs

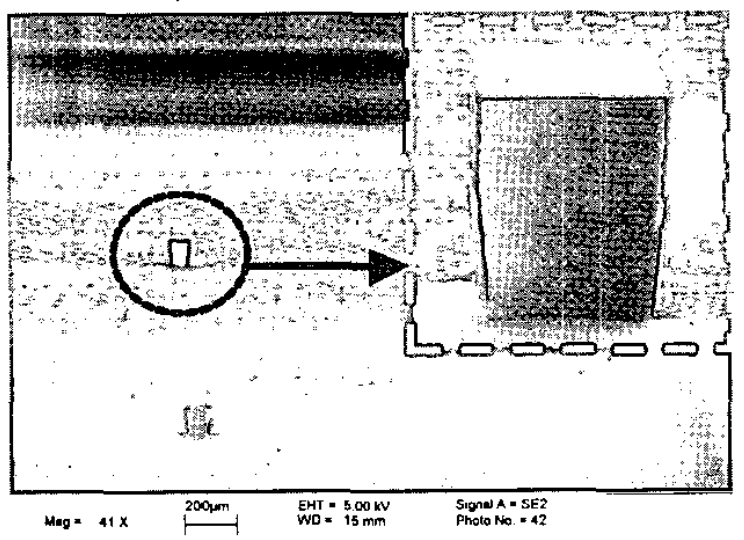

Figure 5. Micropillar structures fabricated on the lid wafer

\section{TRANSDUCERS ' 03}

The 12th International Conference on Solid State Sensors, Actuators and Microsystems, Boston, June 8-12, 2003 
Figure 3 is an SEM microphotograph of batch-fabricated micromirrors produced by this process. A close-up view of tilted comb fingers is presented in Figure 4, showing the precisely aligned vertical comb sets. Figure 5 shows the lid wafer and pillar structures used to deform the microactuators in Fig 3. No damage in the pillars or lid wafer was observed indicating that the lid wafer could be used repeatedly. "

\section{MANUFACTURING ISSUES}

The maximum initial tilt angle that can be formed by the method of plastic deformation that we have described above is limited by the fracture strength of the single- crystal silicon. We have found that $20 \sim 30^{\circ}$ of initial angular displacement is possible with a precise value depending on the specific torsion-bar geometry. There are two ways to control the initial tilting angle; (1) by adjusting the height of the pillar structure, and (2) by changing the position on the mirror where the pillar structure pushes as can be seen in Figure 6.

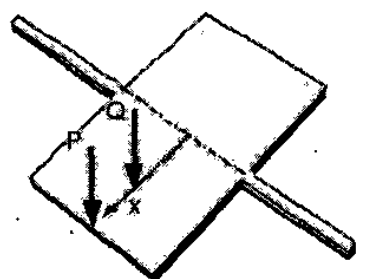

(a)

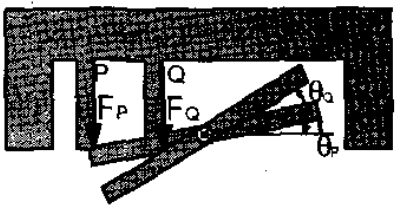

(b)
Figure 6. Different forces applied on the mirror surface depending on the position and height of the pillar structure

Before high-temperature annealing, when the same height of pillar structure is used, forcing the mirror structure at position $Q$ in Fig. 6 causes a larger tilt than if the pillar were at position $P$. The tilt in the second case would, however, be the same if the pillar height were sufficiently increased. Although the tilt angle is the same in these two cases, however, the stresses induced in the torsion bar are not equal. The same angle means the same moment is applied to the torsion bar at position $\mathrm{P}$ and $\mathrm{Q}$, and that is

$$
M_{P}=F_{p} x_{P}=M_{Q}=F_{Q} x_{Q}
$$

and considering the stiffness of torsion bar,

$$
M_{p}=M_{Q}=c \frac{G J \theta}{l}
$$

therefore,

$$
F_{P}=c \frac{G J \theta}{l x_{p}}<F_{Q}=c \frac{G J \theta}{l x_{Q}}
$$

where $G$ is the shear modulus of torsion bar, $J$ is the polar moment-of-inertia of the torsion bar, $l$ is the length of the torsion bar, and $c$ is a constant depending on the crosssectional aspect ratio of the torsion bar.

From Eq. (3), it is obvious that pushing the mirror tip with a longer pillar causes smaller vertical force than pushing the position closer to the torsion bar with a shorter pillar. Because excessive vertical force on the mirror surface may lead not only to the torsion, but also to unwanted bending of the torsion bar or undesirable curvature of the mirror, it is desirable that the pillar structures are designed to be placed at the tip of the mirrors. The measured vertical deflections of torsion bars after plastic deformation were less than $0.3 \mu \mathrm{m}$, and the measured radii-of-curvature of the mirrors were in the order of meters, which indicate there can be only very little plastic deformation induced in the mirror plate.

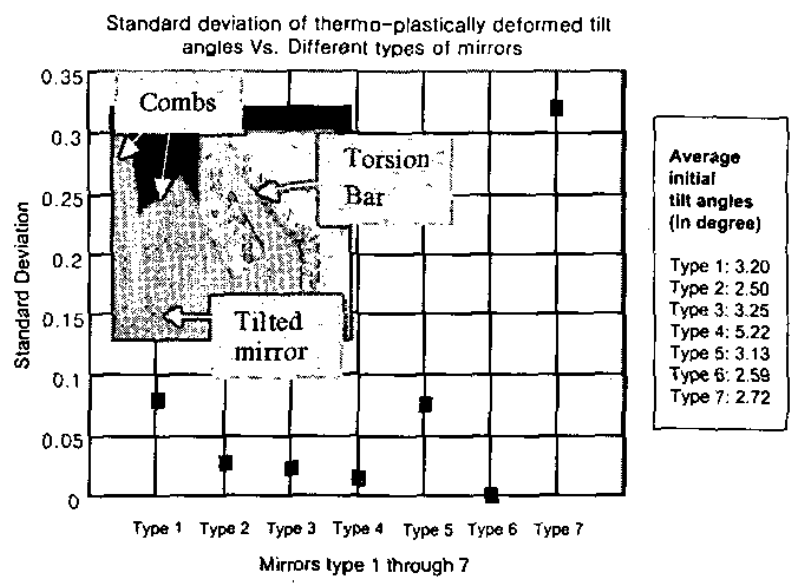

Figure 7. Standard deviation of plastically formed tilting angles for mirrors of different sizes and shapes (Enclosed caption shows the 3-D image generated by WYKO NT3300)

Considering that actuators are made in a batch, the uniformity of the plastic deformations is another parameter to be characterized. The plastically deformed angles of seven different designs were measured and the standard deviations for 10 samples of each type with respect to the average values are plotted in Figure 7. The typical standard deviation is less than $0.1^{\circ}$. Possible sources of deviation are; particles entrapped between the device wafer and lid wafer, or the tolerance for self-alignment between the depression on the device wafer and the protrusion on the lid wafer, which can be easily improved by mating the lid and device wafers with more tight tolerances and in a cleaner environment.

\section{DEVICE CHARACTERIZATION AND DISCUSSION}

The measured resonant frequencies of seven different types of actuators with different sizes of torsion bars, mirrors and comb fingers range from $1.90 \mathrm{kHz}$ to $5.33 \mathrm{kHz}$. The dynamic performance of the type- 4 actuator with the initial tilt angle of $5.22^{\circ}$ is measured and presented in Figure 8. Data were collected using $40 \mathrm{~V}_{\mathrm{dc}}$ and $13 \mathrm{~V}_{\mathrm{ac}}$ drive for the mirror actuator with $50 \mu \mathrm{m}$ thickness of both fixed and moving combs and a mirror size of $800 \mu \mathrm{m} \times 800 \mu \mathrm{m}$. The resonant frequency is measured at $2953 \mathrm{~Hz}$, and a maximum scanning angle of $19.2^{\circ}$ is achieved. The quality factor measured (in air) is 120 .

The plastically deformed angular vertical comb actuator is expected to be exceedingly reliable and stable operationaliy because of its simple and rugged structure made of singlecrystal silicon. To assess its long-term reliability, the actuator was resonated for more than 5 billion cycles using the same driving voltages as are indicated in Fig, 8. The resonant frequency and scanning angle change were measured 
periodically (every 255 million cycles) and these data are presented in Figure 9. The maximum variations of resonant frequency and scanning angle were $0.064 \%$ and $3.6 \%$, respectively. The error bars on the frequency curve are shown since the maximum scanning-angle change was undetectable to $0.2 \mathrm{~Hz}$ variation of the operational frequency.

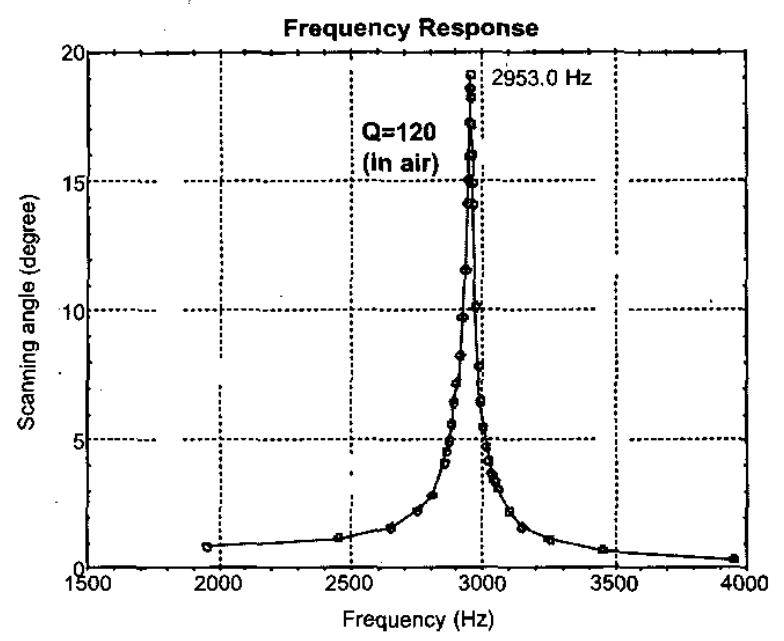

Figure 8. Frequency response of the microactuator

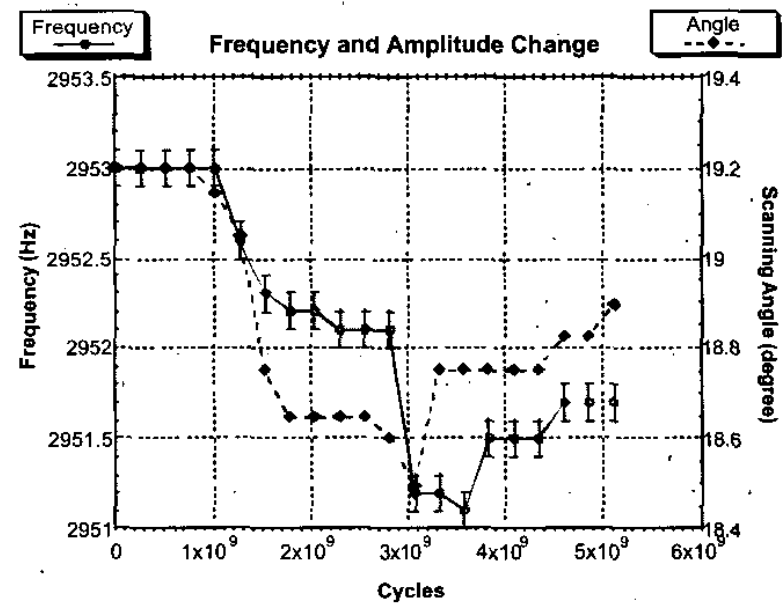

Figure 9. Measured scanning angle and resonant frequency of the microactuator over 5 billion cycles

This experiment was performed with a mirror actuator unpackaged and in the lab. Temperature and particle contaminations, as well as humidity variations are the suspects to cause the slight change of resonant frequency and scanning angle over time rather than the degradation of the plastically deformed flexures. The decrease in frequency as observed in Fig. 9 may possibly result from an increased mirror mass due adhesion of particles from the ambient air as well as from changes in stiffness in the torsion bar.

\section{CONCLUSIONS}

Plastic deformation of silicon was successfully applied to build micro torsional actuators in a straightforward batch process that does not demand any critical alignment steps. The fabrication and characterization of angular vertical combs for scanning-mirror applications have been accomplished and the measured dynamic performance of the actuator was comparable or superior to that reported in earlier verticalcomb actuators. A reliability test on an unpackaged mirror actuated through more than 5 billion cycles of operation showed less than $0.064 \%$ decrease in the resonant frequency:

\section{REFERENCES}

[1] R.A. Conant, P.M. Hagelin, U. Krishnamoorthy, M. Hart, O. Solgaard, K. Y. Lau, R. S. Muller, "A raster-scanning fullmotion video display using polysilicon micromachined mirrors", Sensors \& Actuators, A83, no.1-3, pp.291-296.

[2] V. Milanovic, M. Last, K. S. J. Pister, "Torsional Micromirrors with Lateral Actuators", Transducers 01, Munich, Germany, pp.1290-1301.

[3] D. Hah, S. Huang, H. Nguyen, H. Chang, J.-C. Tsai, M. C. $\mathrm{Wu}$, H. Toshiyoshi, "Low Voltage MEMS Analog Micromirror Arrays with Hidden Vertical Comb-Drive Actuators," 2002 Solid-State Sensor, Actuator and Microsystems Workshop, pp.11-14

[4] J. A. Yeh, H. Jiang, N. C. Tien, "Integrated polysilicon and DRIE bulk silicon micromachining for an electrostatic torsional actuator", Journal of Microelectromechanical Systems, 8, no.4, Dec. 1999, pp.456-465.

[5] R. A. Conant, J. T. Nee, K. Lau, R. S. Mueller "A Flat HighFrequency Scanning Micromirror", 2000 Solid-State Sensor and Actuator Workshop, Hilton Head, SC, pp. 6-9.

[6] U. Krisnamoorthy, O. Solgaard, "Self-aligned Vertical Comdrive Actuators for Optical Scanning Micromirrors", 2001 International Conference on Optical MEMS, p.41.

[7] H. Xie, Y. Pan, G. K. Fedder, "A CMOS-MEMS micromiror with large out-of-plane actuation", 2001 ASME International Mechanical Engineering Congress and Exposition, 3, pp.89-92.

[8] P. R. Patterson; D. Hah, H. Nguyen, H. Toshiyoshi, R.-M. Chao, and M. C. Wu, "A Scanning Micromirror with Angular Comb Drive Actuation", Technical Digest. MEMS 2002 IEEE International Conference. 2002, pp.544-547.

[9] J. R. Patel, A. R. Chaudhuri, "Macroscopic plastic properties of dislocation-free germanium and other semiconductor crystals", Journal of Applied Physics, 34 No.9 1963, pp.2788-2799.

[10] M. M. Farooqui, A. G. R. Evans. "Polysilicon microstructures", Proceedings of the IEEE Micro Electro Mechanical Sy stems. 1991, pp.187-191.

[11] M. A. Huff, A. D. Nikolich, M. A. Schmidt. "A threshold pressure switch utilizing plastic deformation of silicon", TRANSDUCERS '91. 1991 International Conference on Solid-State Sensors and Actuators, pp.177-180.

[12] Y. Fukuta, D. Collard, T. Akiyama, E. H. Yang, H. Fujita, "Microactuated self-assembling of 3D polysilicon structures with reshaping technology", Proceedings IEEE. The Tenth Annual International Workshop on Micro Electro Mechanical Systems. 1997, pp.477-481.

[13] E. Gartner, J. Fruhauf, B. Hannemann, E. Jansch, "Mounting of Si-chips with plastically bent . cantilevers", TRANSDUCERS '01. EUROSENSORS XV. 11th Intemational Conference on Solid-State Sensors and Actuators. 1, 2001, pp.206-209. 